\title{
CBT: helping work out why you feel as you do (formulation) ${ }^{\dagger}$
}

\author{
Lucie Colvin \& Christopher Williams
}

\begin{abstract}
SUMMARY
Cognitive-behavioural therapy (CBT) provides a structure to inform safe and effective practice. It can be helpful for understanding and working with patients, but it can have a wider potential benefit, offering clinicians and teams a self-reflective approach to how they respond to the challenge of working in busy clinical and managerial environments.
\end{abstract}

\section{DECLARATION OF INTEREST}

None

\section{Why complete a formulation in CBT?}

Cognitive-behavioural therapy (CBT) is widely recognised in national treatment guidelines as being an effective form of psychotherapy. It shares with other evidence-based approaches a focus on problems relevant to the person, an underlying structure/model of making sense of experience, and builds on a relationship with the patient (Kuyken 2011). It also provides a helpful personal framework for practitioners to reflect on their own reactions to patients, their team and the everyday challenges they face working in the NHS or in their personal life (Bennett-Levy 2015).

A CBT-based formulation, or clinical summary, is essentially a 'vicious circle' model. It argues that there are two-way relationships between what people think, how they feel emotionally and physically, and what they do (Fig. 1). Crucially, it also incorporates the supports (or not) around the person, together with the practical issues and challenges faced on a daily basis. Each area interacts with the others to affect the person's overall experience. CBT aims to help patients identify and move towards more helpful styles of thinking, behaving or relating to others. Because of the nature of a vicious circle, interventions can be made at any point, and any intervention made can break or improve the overall vicious circle. The aim is also to build on current and past helpful/ adaptive responses and resources.

\section{Using the vicious circle with patients}

Summarising a patient's problems using their own words and sharing this openly and collaboratively empowers them, enabling them to add to or modify their summary of the impact of symptoms. This both provides a model of understanding and identifies clear targets for change. The summary is dynamic and responsive over time, reflecting the progress or worsening of symptoms over a course of treatment.

The formulation also incorporates the support from and problems caused by key people around the person and addresses issues such as isolation or loneliness. It can also include an understanding of our response to the patient and their reactions to us.

\section{Using the approach in our teams and on ourselves}

Whatever their background, all mental health workers assess the impact on the patient of that person's thoughts, feelings, behaviour and relationships. This provides content that can be summarised in a CBT formulation. Thus, a CBT case summary provides a helpful and focused way of communicating key information between team members from different professional backgrounds. It can also be used to inform decision-making.

The CBT-based assessment is also helpful for self-reflection on clinical practice and team working. Critical events cause uncertainty and challenge team morale. Also, certain patients can be unpopular within teams, triggering strong reactions, sometimes including anxiety

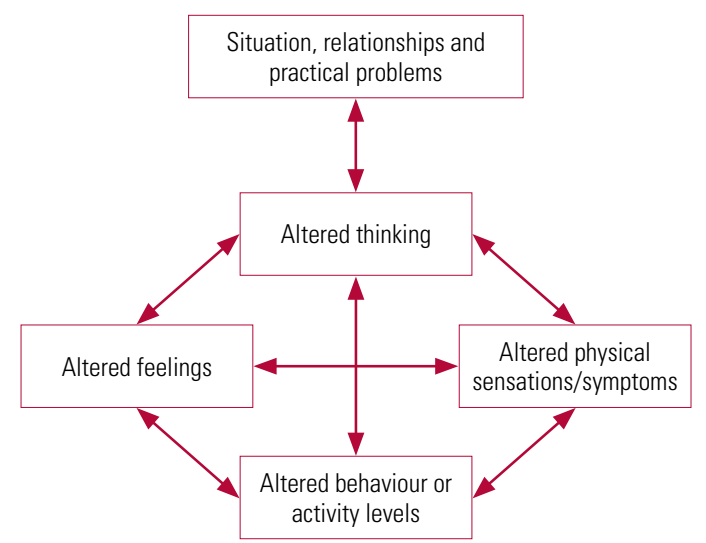

A CBT formulation using the five areas approach (Reproduced, with permission, from Williams 2014).
Lucie Colvin is a consultant psychiatrist in Psychotherapy in Greater Glasgow and Clyde. She is also working towards completing adult psychoanalytic psychotherapy. Chris Williams is Professor of Psychosocial Psychiatry at the University of Glasgow and Presidentelect of the British Association for Behavioural and Cognitive Psychotherapies. He researches models of low-intensity CBT delivery in clinical and community settings. He is author of the popular www. livinglifetothefull.com web course. Correspondence Professor Chris Williams, Institute of Health and Wellbeing, Administration Building $\mathrm{GRH}$, University of Glasgow, Glasgow G12 OXH. UK. Email: chris. williams@glasgow.ac.uk

tThis is the first of a series of refreshments by Chris Williams and colleagues on aspects of cognitivebehavioural therapy. The next will address making effective treatment plans. 
and anger. This can affect us as clinicians and also our colleagues. Examples might be people with personality disorders, multiple comorbid conditions, including drug and alcohol misuse, and those who do not improve with treatment. Unhelpful patterns of thinking and responses can lead to overly strong reactions between colleagues and between teams. Taking a step back to reflect on our own responses in difficult situations and identifying unhelpful patterns of response - can minimise the potential for complaints and also help us to achieve clarity in personal and professional boundaries.

\section{References and further reading}

Bennett-Levy J, Thwaites R, Haarhoff B, et al (2015) Experiencing CBT from the Inside Out: A Self-Practice/Self-Reflection Workbook for Therapists. Guilford Press.

Kuyken W, Padesky C, Dudley R (2011) Collaborative Case Conceptualization: Working Effectively with Clients in CognitiveBehavioral Therapy. Guilford Press

Williams CJ (2014) Overcoming Depression and Low Mood: A Five Areas Approach (4th edn). Taylor \& Francis. 